\title{
Active Hepatitis C Leading to Refractory Thrombotic Thrombocytopenic Purpura - A Dubious Association and The Challenges Faced in Management.
}

\author{
Mounika Gangireddy ${ }^{1}$, Isha Shrimanker ${ }^{1}$, Sandy Saintelia ${ }^{1}$, Vinod K. Nookala ${ }^{1}$ \\ 1. Internal Medicine, University of Pittsburgh Medical Center - UPMC - Pinnacle, Harrisburg, USA
}

Corresponding author: Isha Shrimanker, ims18919@gmail.com

\begin{abstract}
Acquired thrombotic thrombocytopenic purpura is a combination of thrombocytopenia with microangiopathic hemolytic anemia. A 62-year-old female was transferred from an outside hospital for rapidly worsening mental status and severe thrombocytopenia. Laboratory studies were significant for reduced hemoglobin and platelet count along with raised blood urea nitrogen, creatinine, and serum lactate dehydrogenase levels. Peripheral smear showed numerous schistocytes and further testing showed low ADAMTS13 activity, high ADAMTS13 inhibitor, and positive hepatitis $\mathrm{C}$ virus antibody with a high hepatitis $\mathrm{C}$ virus ribonucleic acid (RNA) load. The patient was diagnosed with acquired thrombotic thrombocytopenic purpura and started on plasma exchange and steroids. Since no response was achieved until day four of treatment, weekly rituximab was initiated. After the initial two doses of rituximab, she showed significant improvement clinically. ADAMTS13 levels returned back to normal. Cyclosporine was added, following which platelet counts were normalized. Cyclosporine was discontinued, plasma exchange and steroids were slowly tapered off. Follow-up visits showed that the patient is off treatment and continues to be in remission and on regular treatment for hepatitis C. Acquired thrombotic thrombocytopenic purpura is a hematological emergency. Our patient remained refractory to standard therapies and required rituximab and immunosuppressive agents like cyclosporine. We describe the association of active hepatitis $\mathrm{C}$ with acquired thrombotic thrombocytopenic purpura that was refractory to plasma exchange, high dose steroids and rituximab. As per our knowledge, this is the first case in the literature to describe a possible association between active hepatitis $\mathrm{C}$ and acquired thrombotic thrombocytopenic purpura.
\end{abstract}

Received 06/17/2019

Review began 06/25/2019 Review ended 07/01/2019 Published 07/16/2019

\section{() Copyright 2019}

Gangireddy et al. This is an open access article distributed under the terms of the Creative Commons Attribution License CC-BY 3.0., which permits unrestricted use, distribution, and reproduction in any medium, provided the original author and source are credited.
Categories: Internal Medicine, Pathology, Other

Keywords: refractory thrombotic thrombocytopenic purpura, hepatitis c, rituximab, prednisone, plasma exchange

\section{Introduction}

Thrombotic thrombocytopenic purpura (TTP) is defined as thrombotic microangiopathy that is identified by microangiopathic hemolytic anemia (MAHA), consumptive thrombocytopenia, fever, and systemic involvement, especially renal and neurological abnormalities. The classic 'pentad' of clinical manifestations described previously is present only in 5\% of patients with TTP [1]. In 1924, Eli Moschcowitz first reported the occurrence of TTP in a 16-year-old girl with symptoms of fever, petechiae and neurological involvement along with thrombocytopenia and hemolytic anemia [2].

TTP occurs due to a decrease in the activity of ADAMTS13, which is a von Willebrand factor (vWF)-cleaving protease. Reduced activity of this enzyme results in an aggregation of unusually large vWF multimers. In the presence of shear stress, these uncleaved multimers of vWF result in adhesion of platelets to the damaged endothelial wall of the blood vessel and thus lead to platelet aggregation. This results in the formation of platelet-rich microthrombi that causes occlusion of the vasculature, eventually causing MAHA, thrombocytopenia and visceral ischemia.

The two forms of TTP include hereditary and acquired. The hereditary form is characterized by the presence of mutated genes of ADAMTS13. The acquired form, on the other hand, occurs due to autoantibody formation against ADAMTS13 resulting in reduced activity. Autoimmunity is hypothesized to be secondary to neutralizing antibodies that suppress the proteolytic activity of ADAMTS13 or due to the nonneutralizing antibodies that result in interference with the binding of ADAMTS13 to endothelial surfaces or result in increased ADAMTS13 clearance [3].

The 2012 American Society of Apheresis Consensus Conference on TTP described remission when a platelet count $>150000 / \mu \mathrm{L}$ is present for two consecutive days, lactate dehydrogenase (LDH) that is within the normal range and gradual improvement in neurologic symptoms [4]. The mainstay treatment of TTP involves plasma exchange (PLEX) and corticosteroids. 
In $>90 \%$ of patients, TTP can be fatal if no treatment is received [5]. Refractory TTP is described as a failure to achieve remission in 4-7 days despite initiation of plasma exchange and steroid therapy or gradual worsening of the clinical condition. There is limited literature stating the management of TTP.

\section{Case Presentation}

A 62-year-old African American female with a past medical history of hypertension and heavy alcohol abuse was transferred from an outside hospital with worsening mental status and severe thrombocytopenia. Vital signs at the time of admission showed a pulse of 93 beats per minute, blood pressure of $188 / 114 \mathrm{~mm}$ of $\mathrm{Hg}$, respiratory rate of 16 breaths per minute and was saturating $100 \%$ on room air. She was awake but oriented only to self. The rest of the physical examination was unremarkable.

Laboratory studies revealed severe thrombocytopenia of $21,000 / \mu \mathrm{L}$, low hemoglobin of $8.7 \mathrm{gm} / \mathrm{dl}$, and hematocrit of $23.7 \%$ with a normal white cell count. The renal panel revealed an elevated blood urea nitrogen of $54 \mathrm{mg} / \mathrm{dl}$ and creatinine of $2.64 \mathrm{mg} / \mathrm{dl}$. The hepatic panel showed alanine aminotransferase of 78 $\mathrm{U} / 1$, aspartate aminotransferase of $221 \mathrm{U} / 1$ and alkaline phosphatase of $60 \mathrm{U} / 1$. She had elevated indirect bilirubin with negative Coombs test, reticulocyte count was elevated at 4.2\%, LDH of $3643 \mathrm{U} / 1$ and very low haptoglobin $<5.8 \mathrm{mg} / \mathrm{dl}$. Coagulation panel revealed a normal partial thromboplastin time, prothrombin time and reduced fibrinogen levels. Peripheral smear demonstrated numerous schistocytes with polychromasia and severe thrombocytopenia, indicative of microangiopathic hemolysis. Imaging included computed tomography of chest, abdomen, and pelvis which was unremarkable. The activity of vWF protease was severely low at $<3 \%$ whereas vWF protease inhibitor was high at $2.9 \mathrm{BEU}$ at the time of diagnosis. Further testing included a hepatitis panel which revealed positive hepatitis $\mathrm{C}$ virus (HCV) antibody with polymerase chain reaction (PCR) for HCV ribonucleic acid (RNA) showing a viral load of 5,850,000 IU/mL.

At this point, the patient was diagnosed with acquired TTP most likely related to the active hepatitis C infection. Immediately started on PLEX with increased volume of fresh frozen plasma (around 1.5 times the body volume) and prednisone at $1 \mathrm{mg} / \mathrm{kg}$ daily. Platelet counts were $23,000 / \mu \mathrm{L}$ on day four of plasma exchange and prednisone. TTP was then considered to be refractory and immunosuppressive therapy with weekly rituximab $375 \mathrm{mg} / \mathrm{m} 2$ was started along with plasma exchange. Around the same time, her respiratory status worsened due to pneumonia, which required intubation. She showed quick recovery from the infection and was extubated within 72 hours. She showed clinical improvement by the second week of hospitalization. After a week of rituximab administration, platelet count improved to $78,000 / \mu \mathrm{L}$ along with $\mathrm{LDH}$ and reticulocyte count. Peripheral smear revealed the presence of fewer schistocytes. After two weeks of treatment with rituximab, ADAMTS13 activity returned to normal with undetectable vWF inhibitor activity (Figure 1). After completion of four weeks of rituximab, an improvement was seen in the platelet count to $89,000 / \mu \mathrm{L}$ and hematocrit to $28.3 \%$. Peripheral smear showed few schistocytes. PLEX was then switched from daily to three times a week. She responded to rituximab as evidenced by the appearance of fewer schistocytes in the peripheral smear but not completely as platelet counts continued to remain low (Figure 2). She was then started on the immunomodulatory agent, cyclosporine $50 \mathrm{mg}$ twice daily and two weeks later the platelet count improved to 220,000/ $\mu \mathrm{L}$. Cyclosporine was discontinued after three weeks and the platelet count returned to 250,000/ $\mathrm{LL}$ (Figure 3).

140

120

100

80

60

40

20

0

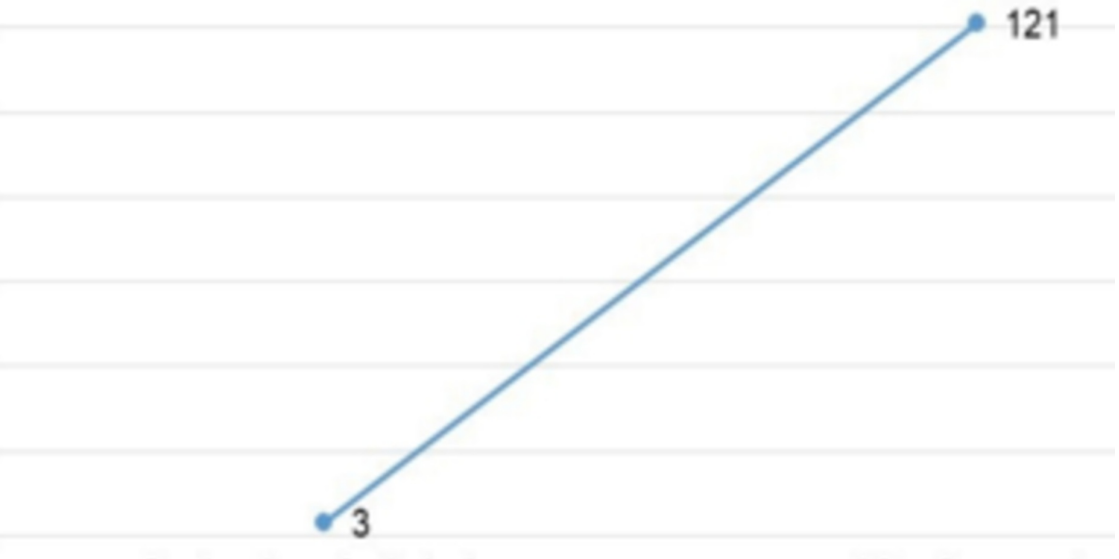

On the day of admission

After four weeks

ADAMTS13\% activity

FIGURE 1: Trend of ADAMTS13 in a duration of one month

Rituximab was given around three weeks. ADMAST13 level was drawn after a week of the first dose of rituximab. 


\section{Cureus}
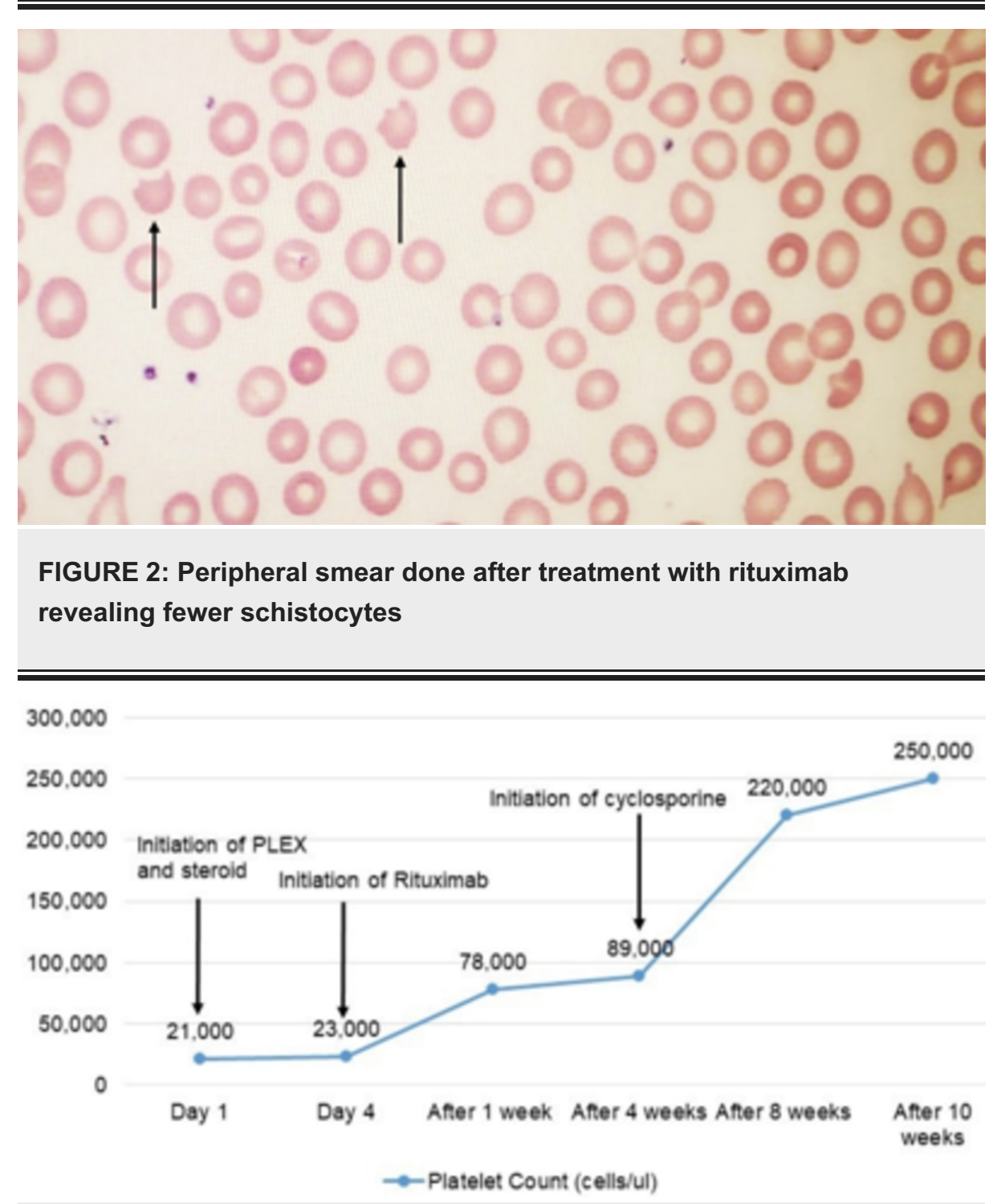

\section{FIGURE 3: Trends of platelet count over ten weeks}

Improvement in platelet count was seen after treatment with cyclosporine

PLEX: Plasma exchange

In total, she received a total of 70 days of PLEX sessions. Follow-up visits revealed that platelet counts, renal function, LDH, reticulocyte count, hemoglobin, and hepatic function panel were all within normal limits. Currently, she is in complete remission and is off treatment. The patient regularly follows up for the management of hepatitis $\mathrm{C}$ infection.

\section{Discussion}

Diagnosis of MAHA including TTP is mainly through clinical evaluation, routine laboratory studies and schistocytes on peripheral smear. Our patient was diagnosed with acquired TTP due to her altered mental status along with microangiopathic hemolytic anemia by the presence of schistocytes on peripheral smear, marked thrombocytopenia, high serum LDH and creatinine levels. A decreased ADAMTS13 activity (<3\%) and increased ADAMTS13 inhibitor levels (2.9 BEU) further confirmed the diagnosis of TTP, ruling out other causes of thrombocytopenia and MAHA [6].

Infections of bacterial, viral and fungal origin have been reported to precipitate the development of TTP [7]. Viral hepatitis was described as one of the risk factors for TTP [8]. The current serological assays used to identify HCV antibodies cannot definitively distinguish between acute and chronic HCV infection. This is 
attributed to the fact that viral markers such as anti-HCV immunoglobulin $g$ (IgG) antibody, and HCV RNA may be found in both acute and chronic phase of HCV infection. Clinicians conventionally differentiate acute and chronic HCV infection on the basis of risk factors, clinical presentation, and history of seronegative markers in the past [9-10]. Our patient did not undergo any serological testing in the past and was diagnosed with active hepatitis $\mathrm{C}$ infection due to the presence of positive HCV antibody with PCR for HCV RNA showing a viral load of 5,850,000 IU/mL. The serological markers for human immunodeficiency virus (HIV) antibody, total hepatitis B core antibody IgM, hepatitis B surface antigen were all non-reactive.

Current literature identifies the association of chronic hepatitis $\mathrm{C}$ and interferon therapy with the development of TTP. lyoda et al. reported a case of TTP that developed during interferon (IFN) therapy in the 16th week, whereas Kitano et al. reported the development of TTP after a month's therapy with IFN [11$12]$.

Kamal et al., reported a case of acute hepatitis C that manifested as MAHA resembling TTP and showed resolution of symptoms once the viral infection settled down [13]. We report a case of active hepatitis C associated with serologically proven TTP, which to our knowledge is the first of its kind.

Standard treatment available for TTP is PLEX and corticosteroids. PLEX should be started immediately while awaiting the confirmatory laboratory results. Our patient was started on PLEX and prednisone immediately after peripheral smear showed schistocytes. Equal volumes of fresh frozen plasma are used for PLEX but volumes can be increased to 1.5 times if there is no response or if clinically deteriorating [6].

Our patient was described as having refractory TTP since no response was achieved after treatment with high dose steroids and PLEX after four days of therapy. In the majority of the literature, refractory TTP is described when a patient fails to improve clinically even after standard treatment or failure to normalize platelet count even after 4-7 days of PLEX. The incidence rate of patients with refractory TTP and in need of further management has been reported to be between 10-42\% [14]. In such conditions, there is a need to reevaluate any other causes for thrombocytopenia or anemia. Our patient, however, did not have any obvious cause which could explain the low platelet count.

Rituximab is a monoclonal antibody that targets the B lymphocytes, specifically the CD20 antigen. Literature states that the use of rituximab reduces the duration to achieve response to platelet count, helps to improve ADAMTS13 activity, decreases the extent of PLEX and helps to prevent relapses. After the initiation of rituximab with a dose of $375 \mathrm{mg}$ per week for a total of four weeks, which is the most commonly used dose, her clinical manifestations ameliorated but there was a failure to achieve the desired platelet level [6].

Treatment with immunomodulatory agents can be considered if refractory TTP persists even after treatment with rituximab [6]. Cyclosporine prevents the activation of T-cell, thus causing inhibition of interleukin-2 (IL-2) receptor expression and eventually decreased the production of IL-2. Cataland et al. revealed that the use of cyclosporine along with PLEX helps in improving ADAMTS13 activity and reducing the activity of ADAMTS13 inhibitor. Out of all the patients involved, 89\% achieved remission, proving the efficacy of cyclosporine in refractory cases of TTP as well [15]. Our patient showed significant improvement with combined cyclosporine and PLEX therapy along with steroid administration (Figure 4). 


\section{Cureus}

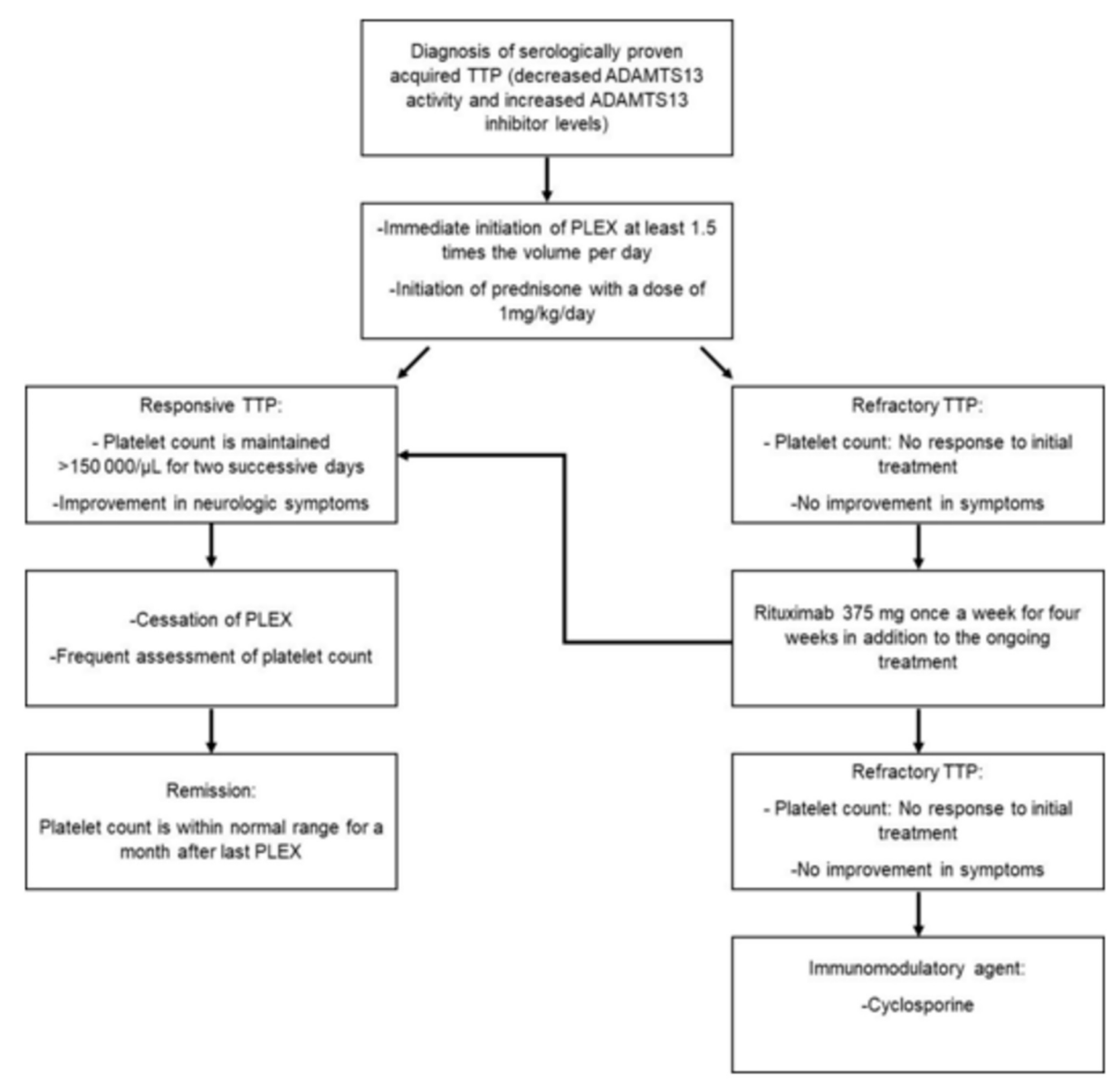

\section{FIGURE 4: Our approach to the management of refractory TTP}

Our patient remained refractory to standard therapies like PLEX and steroid administration. Improvement was seen with rituximab and immunosuppressive agents like cyclosporine.

TTP: Thrombotic thrombocytopenic purpura; PLEX: Plasma exchange

Other treatment options available for refractory TTP include immunosuppressive treatment with cyclophosphamide and vincristine individually as well as in combination. Splenectomy has also been considered as a treatment modality, but data to prove its effectiveness is limited [6].

Recent advances in the field of management for refractory TTP have been reassuring. N-acetylcysteine (NAC) is primarily used in acetaminophen toxicity or in respiratory diseases to reduce the mucinous secretions. vWF is structurally similar to mucin and just like NAC is used to cleave the disulfide bonds in multimers of mucin, it is also postulated that NAC can be used as a replacement to ADAMTS13 and cleave vWF multimers [16]. The usefulness of NAC has been described in the case report by Li et al. involving the treatment of refractory TTP, however, clinical trials are pertinent to prove its clinical efficacy [17].

Bortezomib, which is a proteasome inhibitor has reportedly helped to reduce the ADAMTS13 inhibitor levels in refractory TTP. Shortt et al. reported the case of a 53-year-old woman who was diagnosed with TTP unresponsive to prednisone, PLEX, rituximab, cyclophosphamide, and NAC, but showed improvement after treatment with bortezomib [18].

Eculizumab which is a monoclonal antibody has been effective in treatment of complement-mediated thrombotic microangiopathy [19]. However, its role in treatment of acquired TTP has not been established.

The major pitfall with these therapies is that there is limited data to prove their effectiveness and hence more research is needed in this arena.

\section{Conclusions}

The etiology of TTP is still an enigma in the field of hematology. More extensive studies need to be performed to prove the association between hepatitis $\mathrm{C}$ infection and TTP. It is still debatable whether 
treating hepatitis $\mathrm{C}$ infection would prevent further relapses of TTP. In conclusion, timely and appropriate management is required in patients with refractory TTP to prevent fatal consequences.

\section{Additional Information \\ Disclosures}

Human subjects: Consent was obtained by all participants in this study. UPMC Pinnacle Institutional Review Board issued approval NA. As long as no personal identifiable information is used and all information presented for publication is de-identified per HIPAA regulations, this research is exempt under 45 CFR 46.101 (b) (4) and does not need to be submitted to the IRB for review and approval. Conflicts of interest: In compliance with the ICMJE uniform disclosure form, all authors declare the following: Payment/services info: All authors have declared that no financial support was received from any organization for the submitted work. Financial relationships: All authors have declared that they have no financial relationships at present or within the previous three years with any organizations that might have an interest in the submitted work. Other relationships: All authors have declared that there are no other relationships or activities that could appear to have influenced the submitted work.

\section{References}

1. George JN: How I treat patients with thrombotic thrombocytopenic purpura . 2010. Blood. 2010, 116:40604069. 10.1182/blood-2010-07-271445

2. Moschcowitz E: An acute febrile pleiochromic anemia with hyaline thrombosis of the terminal arterioles and capillaries: an undescribed disease. JAMA Intern Med. 1925, 36:89-93. 10.1001/archinte.1925.00120130092009

3. Scheiflinger F, Knobl P, Trattner B, et al.: Nonneutralizing IgM and IgG antibodies to von Willebrand factorcleaving protease (ADAMTS-13) in a patient with thrombotic thrombocytopenic purpura. Blood. 2003, 102:3241-3243. 10.1182/blood-2003-05-1616

4. Sarode R, Bandarenko N, Brecher ME, Kiss JE, Marques MB, Szczepiorkowski ZM, Winters JL: Thrombotic thrombocytopenic purpura: 2012 American Society for Apheresis (ASFA) consensus conference on classification, diagnosis, management, and future research. J Clin Apher. 2014, 29:148-167. 10.1002/jca.21302

5. Hand JP, Lawlor ER, Yong CK, Davis JH: Successful use of cyclosporine A in the treatment of refractory thrombotic thrombocytopenic purpura. Br J Haematol. 1998, 100:597-599. 10.1046/j.13652141.1998.00602.x

6. Sayani FA, Abrams CS: How I treat refractory thrombotic thrombocytopenic purpura. Blood. 2015, 125:38603867. 10.1182/blood-2014-11-551580

7. Douglas KW, Pollock KG, Young D, Catlow J, Green R: Infection frequently triggers thrombotic microangiopathy in patients with preexisting risk factors: a single-institution experience. J Clin Apher. 2010, 25:47-53. 10.1002/jca.20226

8. Booth KK, Terrell DR, Vesely SK, George JN: Systemic infections mimicking thrombotic thrombocytopenic purpura. Am J Hematol. 2011, 86:743-751. 10.1002/ajh.22091

9. Araujo AC, Astrakhantseva IV, Fields HA, Kamili S: Distinguishing acute from chronic hepatitis C virus (HCV) infection based on antibody reactivities to specific HCV structural and nonstructural proteins. J Clin Microbiol. 2011, 49:54-57. 10.1128/jcm.01064-10

10. McGovern BH, Birch CE, Bowen MJ, Reyor LL, Nagami EH, Chung RT, Kim AY: Improving the diagnosis of acute hepatitis C virus infection with expanded viral load criteria. Clin Infect Dis. 2009, 49:1051-1060. $10.1086 / 605561$

11. Iyoda K, Kato M, Nakagawa T, et al.: Thrombotic thrombocytopenic purpura developed suddenly during interferon treatment for chronic hepatitis C. J Gastroenterol. 1998, 33:588-592. 10.1007/s005350050139

12. Kitano K, Gibo Y, Kamijo A, et al.: Thrombotic thrombocytopenic purpura associated with pegylatedinterferon alpha-2a by an ADAMTS13 inhibitor in a patient with chronic hepatitis C. Haematologica. 2006, $91: 34$.

13. Kamal A, Hafez Youssef A, Refaat Mansour A: Acute hepatitis caused by genotype 4 HCV presenting with microangiopathic hemolytic anemia. ACG Case Rep J. 2018, 5:73. 10.14309/cri.2018.73

14. Blitzer JB, Granfortuna JM, Gottlieb AJ, et al.: Thrombotic thrombocytopenic purpura: treatment with plasmapheresis. Am J Hematol. 1987, 24:329-339. 10.1002/ajh.2830240402

15. Cataland SR, Jin M, Lin S, Kennedy MS, Kraut EH, George JN, Wu HM: Cyclosporin and plasma exchange in thrombotic thrombocytopenic purpura: long-term follow-up with serial analysis of ADAMTS13 activity. Br J Haematol. 2007, 139:486-493. 10.1111/j.1365-2141.2007.06819.x

16. Chen J, Reheman A, Gushiken FC, et al.: N-acetylcysteine reduces the size and activity of von Willebrand factor in human plasma and mice. J Clin Invest. 2011, 121:593-603. 10.1172/jci41062

17. Li GW, Rambally S, Kamboj J, Reilly S, Moake JL, Udden MM, Mims MP: Treatment of refractory thrombotic thrombocytopenic purpura with N-acetylcysteine: a case report. Transfusion. 2014, 54:1221-1224. 10.1111/trf.12440

18. Shortt J, Oh DH, Opat SS: ADAMTS13 antibody depletion by bortezomib in thrombotic thrombocytopenic purpura. N Engl J Med. 2013, 368:90-92. 10.1056/NEJMc1213206

19. Waters AM, Licht C: aHUS caused by complement dysregulation: new therapies on the horizon . Pediatr Nephrol. 2011, 26:41-57. 10.1007/s00467-010-1556-4 